Published in "Gait \& Posture 42(4): 419-423, 2015"

which should be cited to refer to this work.

\title{
Bioceramic fabrics improve quiet standing posture and handstand stability in expert gymnasts
}

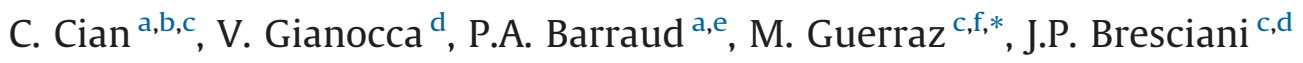 \\ a Institut de Recherche Biomédicale des Armées, 91223 Brétigny sur Orge, France \\ ${ }^{\mathrm{b}}$ Univ Grenoble Alpes, LPNC, F-38000 Grenoble, France \\ ' CNRS, LPNC, F-38000 Grenoble, France \\ ${ }^{\mathrm{d}}$ University of Fribourg, Department of Medicine, Fribourg, Switzerland \\ ${ }^{\text {e }}$ CNRS, TIMC-IMAG, F-38000 Grenoble, France \\ ${ }^{\mathrm{f}}$ Univ Savoie Mont Blanc, LPNC, F-73000 Chambéry, France
}

Bioceramic fabrics have been claimed to improve blood circulation, thermoregulation and muscle relaxation, thereby also improving muscular activity. Here we tested whether bioceramic fabrics have an effect on postural control and contribute to improve postural stability. In Experiment 1, we tested whether bioceramic fabrics contribute to reduce body-sway when maintaining standard standing posture. In Experiment 2, we measured the effect of bioceramic fabrics on body-sway when maintaining a more instable posture, namely a handstand hold. For both experiments, postural oscillations were measured using a force platform with four strain gauges that recorded the displacements of the center of pressure $(\mathrm{CoP})$ in the horizontal plane. In half of the trials, the participants wore a full-body second skin suit containing a bioceramic layer. In the other half of the trials, they wore a 'placebo' second skin suit that had the same cut, appearance and elasticity as the bioceramic suit but did not contain the bioceramic layer. In both experiments, the surface of displacement of the CoP was significantly smaller when participants were wearing the bioceramic suit than when they were wearing the placebo suit. The results suggest that bioceramic fabrics do have an effect on postural control and improve postural stability.

\section{Introduction}

Bioceramic materials, materials that emit high-performance far-infrared (FIR) rays, have recently been the object of high interest mainly for therapeutic purposes including healthpromoting practices [1]. Bioceramic garments may also be manufactured for performance enhancing apparel in both leisure activities and competitive sports area [2]. For Yoo et al. [3], ceramics increase thermal insulation by reflecting FIR rays from the body back to the body surface. The principle source of energy needed to power the FIR emission from the garments comes from the human body. Energy from the human body is transferred to these ceramic particles which are acting as "absorbers", maintain

\footnotetext{
* Corresponding author at: Laboratory of Psychology and NeuroCognition, UMR 5105 CNRS - University Savoie Mont Blanc, BP 1104, 73011 Chambery Cedex, France. Tel.: +33 4797591 86; fax: +33 0479758599 .

E-mail address: Michel.guerraz@univ-savoie.fr (M. Guerraz).
}

their temperature at sufficiently high levels and then emit FIR back to the body [2]. The body can experience energy of FIR as a radiant heat which can penetrate up to $4 \mathrm{~cm}$ beneath the skin. Elevation of skin temperature observed suggests that this might result of the acceleration of percutaneous blood circulation [3]. Enhancement of skin microcirculation has been shown using FIR in an animal model [4]. In line with this, an increase in forearm blood flow has been observed in resting subjects whose left arms were in fabric ribbon which was lined with ceramic disks [5]. Gloves have been made out of FIR emitting fabrics and there have been reports that these gloves can be used to treat Raynaud's syndrome [6], notably via improved vasodilatation and circulation. Significant improvements were also documented in both subjective measures of pain and discomfort and in objective measures of temperature and grip [6].

The usefulness of bioceramic materials added into fabrics has also been shown during physical exercise. For instance, ceramic coated clothing increased blood flow during a 30 min exercise on a bicycle ergometer at a work rate of $75 \mathrm{~W}$ in a cool environment 
[7]. Moreover, there were tendencies toward decreased tiredness and reduced skin temperature in subjects wearing a bioceramic shirt while running $30 \mathrm{~min}$ at a steady velocity of $6 \mathrm{~km} / \mathrm{h}$; [1]. Leung et al. [8] used electro-stimulation of amphibian skeletal muscle and found that FIR emitting ceramics delayed the onset of fatigue induced by muscle contraction. They also suggested that the presence of bioceramics could normalize acidification following muscle contractions. Even though the exact mechanisms of the hyperthermic effects and biological activities of FIR irradiation are still poorly understood, converging evidence suggests that bioceramic materials contribute to increase fatigue resistance, decrease energy expenditure even during steady state [1] and, improve dexterity [6].

Physical but also normal everyday activities depend to a large extent on the ability to control our balance. Postural equilibrium involves actively maintaining the chosen body configuration against gravity, and internal or external disturbances [9]. It therefore constitutes an important attribute of the musculoskeletal-proprioceptive apparatus with skeletal muscles continually making fine adjustments that hold the body in quasi-stationary positions. Any way to improve physical performance, dexterity and muscle fatigue, might therefore significantly affect postural performance. Therefore, bioceramic garments might well contribute to improve postural control, which to the best of our knowledge has never been investigated. In the present experiments, we tested whether bioceramic fabrics affect postural stability during unperturbed standing in non-athlete participants and for maintaining a handstand hold in expert gymnasts.

\section{Materials and methods}

\subsection{Participants}

Twelve participants participated in Experiment 1 (aged 20-35, mean $=28.5,6$ males). Fourteen confirmed gymnasts (nationally ranked in Switzerland, having more than 10 years of experience in gymnastics competition at the regional level or higher) participated in Experiment 2 (aged 15-28, mean $=21.5,9$ males). These participants were selected for their ability to maintain/ sustain a handstand hold for at least $5 \mathrm{~s}$. Participants had no history of balance or neuromuscular disorder. They were naïve to the aim of the study and gave informed-written consent prior to the study. The research was performed in accordance with the ethical standards specified by the 1964 Declaration of Helsinki and approved by the ethics review board of the University of Fribourg.

\subsection{Apparatus}

Both experiments took place in a well-lit room. A force platform equipped with four strain gauges linked to a computer was used to record the displacements of the center of feet (Experiment 1 ) or hand (Experiment 2) pressure (CoP) in the horizontal plane (i.e., along the $X$ - and $Y$-axis). The force-platform was developed in the Biomedical Research Institute of French Armed Forces (IRBA). It is made of two steel plates $(50 \mathrm{~cm} \times 50 \mathrm{~cm} \times 3 \mathrm{~cm}$ ) and has a total height of $18 \mathrm{~cm}$. The upper plate lies on four strain gauges (AG50C3SH10ef SCAIME) close to the vertices of the plate and distant from each other by $40 \mathrm{~cm}$. The mean resolution for a $70 \mathrm{~kg}$ subject standing in the centre of the platform is inferior to $0.1 \mathrm{~mm}$. The sampling frequency of the platform is $100 \mathrm{~Hz}$, and high frequencies were attenuated using a second-order low-pass filter with a $10 \mathrm{~Hz}$ cut-off frequency. The antero-posterior (AP) and medio-lateral (ML) axes are referenced to the force platform.

Two types of suit were worn by the participants during the experiment: Bioceramic and Placebo suits. Both types of suit were made of polyester (85\%) and spandex (15\%) in addition to which bioceramic suits also integrated a bioceramic induction/layer ( $7 \mathrm{~g}$ of bioceramic $/ \mathrm{m}^{2}$ of cloth, i.e., $5 \%$ of total fabrics' weight). All suits consisted of second-skin, two-piece (top and bottom) black suits covering the whole body from neck to wrists and ankles. The suits came in four different sizes (S, M, L and XL) and the size worn by any given participant was always the same for the bioceramic and placebo suits. For any given size, bioceramic and placebo suits were identical in size, appearance and elasticity (220\%). The only difference between bioceramic and placebo suits was a garment label indicating A or B.

\subsection{Task}

Experiment 1: Participants were instructed to stand quiet on the force platform with arms relaxed on the side of the body while fixing a plumb line located $90 \mathrm{~cm}$ in front of them for the $60 \mathrm{~s}$ duration of the trial. They stood barefoot on the platform with their feet abducted at $30^{\circ}$ and heels separated by $2 \mathrm{~cm}$.

Experiment 2: At the beginning of each trial, the gymnasts got up into a handstand on the force platform. An experimenter helped them establish and stabilize a proper handstand position. Once the gymnasts felt they were comfortable and stable in this position, the experimenter released their legs. Their task was to maintain the position and try to sway as little as possible for the $5 \mathrm{~s}$ duration of the trial. At the end of the trial, the experimenter took hold of their legs and helped bringing them back on the floor. For the handstand, each gymnast was free to use the hand width that was the most comfortable for him/her. But they were then required to use the same hand width and position throughout the experiment. Fig. 1 shows a participant performing the task (i.e., maintaining a handstand) with one of the experimental suits.

\subsection{Design}

Experiment 1: Participants performed four trials per suit type, for a total of 8 trials. Each trial lasted $60 \mathrm{~s}$ with a 1 min rest break
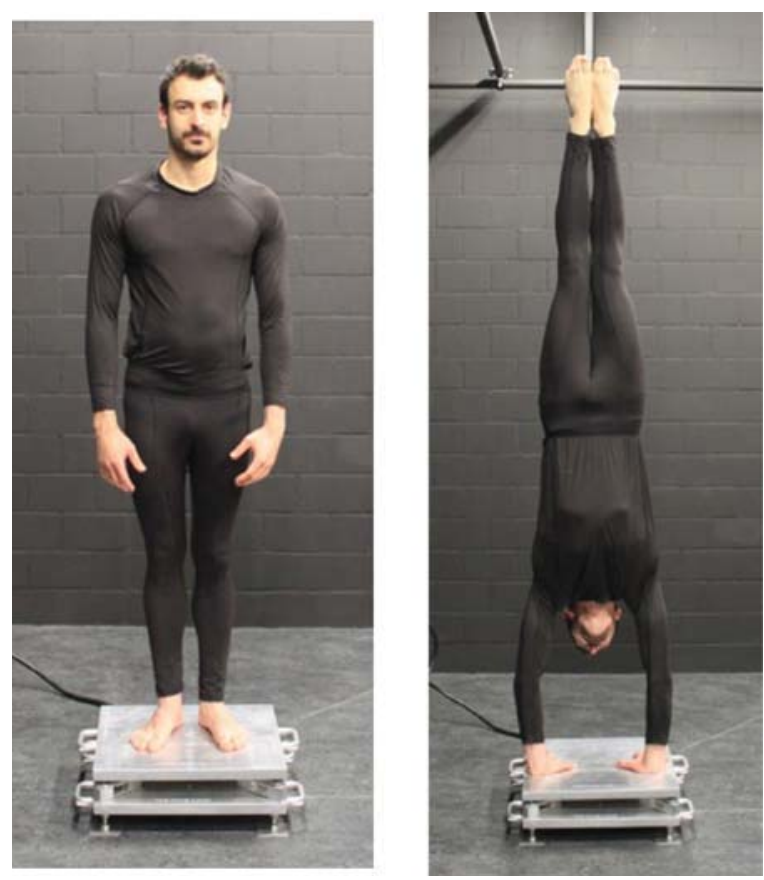

Fig. 1. A participant wearing the bioceramic suit while performing the standing (Experiment 1, left image) and the handstand (Experiment 2, right image) postural tasks. Different participants participated in the two experiments. 
between two successive trials. Participants also got a 10 min break to change suit after four trials were performed with the first suit type. Half of the participants started with the bioceramic suit, and the other half with the placebo suit.

Experiment 2: Gymnasts performed five trials per suit type, for a total of ten trials. Each trial lasted $5 \mathrm{~s}$ with a $1 \mathrm{~min}$ rest break between two successive trials. After five trials were performed with one suit type, the gymnasts had a 10 min break during which they changed suit before completing a second series of five trials with the second suit types (still with $1 \mathrm{~min}$ rest between each trial). To avoid any fatigue-related bias in the results, half of the gymnasts (seven) started the experiment wearing the bioceramic suit, and the other half wearing the placebo suit.

A double-blind procedure was used for both experiments. Neither the participants nor the experimenters did know which of the A and B suits corresponded to bioceramic and placebo, respectively. The experiments lasted about 45 min each including instructions.

\subsection{Data analysis}

For each trial, six parameters were calculated from the CoP coordinates $(X$ and $Y)$ : ( 1 ) length of sway path $(\mathrm{mm})$, i.e., the total distance travelled by the CoP over the trial period, 2 ) area of the 95\% confidence ellipse $\left(\mathrm{mm}^{2}\right)$, ( 3 and 4 ) antero-posterior (AP) and medio-lateral $(\mathrm{ML})$ range of motion of the $\mathrm{CoP}(\mathrm{mm})$, i.e., the difference between the two extreme position values of the CoP in the respective directions, and (5 and 6) velocity ( $\mathrm{mm} / \mathrm{s}$ ) for AP and ML motion of the CoP. Movements of the CoP were calculated with Matlab 7.8 (MathWorks) following to the recommendations of Schubert et al. [10].

For each participant, suit condition, and dependent variable, the mean value and the standard deviation of the mean were computed. For each dependent variable, the resulting values in the two suit conditions were compared (bioceramic suit vs placebo suit) using paired t-tests when all assumptions of parametric data were met, and using Wilcoxon signed-rank tests otherwise. These tests allowed us to assess whether the two types of suit affected differently the average body-sway but also bodysway variability.

\section{Results}

\subsection{Experiment 1}

Length: The sway path of the CoP was significantly shorter when participants were wearing the bioceramic suit (mean length of the path $=602 \mathrm{~mm}$ ) than when they were wearing the placebo suit (mean length of the path $=632 \mathrm{~mm}, t(11)=-4.379, p=.0011$, $r=-.80$, see Fig. $2 \mathrm{~A}$ ). Individual sway path variability (intrasubject) was not affected by the suit type.

Area of the 95\% confidence ellipse: As can be seen in Fig. 2B, the surface of displacement of the CoP was significantly smaller when participants were wearing the bioceramic suit (mean area $=192 \mathrm{~mm}^{2}$ ) than when they were wearing the placebo suit ( mean area $=231 \mathrm{~mm}^{2}$ ), $t(11)=-2.935, p=.021, r=.66$. Individual surface variability (intra-subject) was unaffected by the suit type.

Range: Neither the antero-posterior nor the lateral range did significantly differ between the two suit conditions. Individual range variability was not altered either by the suit type.

Velocity: The mean velocity of antero-posterior displacements of the CoP was significantly smaller with the bioceramic ( mean $=7.40$, $\mathrm{SD}=1.65$ ) than with the placebo suit (mean $=7.84, \mathrm{SD}=1.68$ ), $t(11)=-4.62, p=.0007, r=.81$. There was also a difference in lateral sway velocity between bioceramic (mean $=5.29, \mathrm{SD}=1.12$ ) and placebo suits (mean $=5.48, S D=1.29$ ), but it failed to reach significance $(t(11)=-1.84, p=.09, r=.49)$. Individual velocity variability remained unaffected by the suit type.

\subsection{Experiment 2}

Length: The sway path of the CoP was shorter with the bioceramic suit (mean $=535.6 \mathrm{~mm}$ ) than with the placebo suit ( mean $=569.5 \mathrm{~mm}$ ), indicating that participants swayed less when wearing the bioceramic suit. This difference failed to reach significance, $t(13)=-1.816, p=.0924$, even though the effect size was fairly substantial, $r=.45$. Individual sway path variability did not differ significantly between the two suit conditions.

Area of the $95 \%$ confidence ellipse: The surface of displacement of the CoP was significantly smaller with the bioceramic suit (mean area $=1941 \mathrm{~mm}^{2}$ ) than with the placebo suit (mean area $=$ $2667 \mathrm{~mm}^{2}$ ), $Z=5, p=.0012, r=-.61$ (see Fig. 3A). The same
A

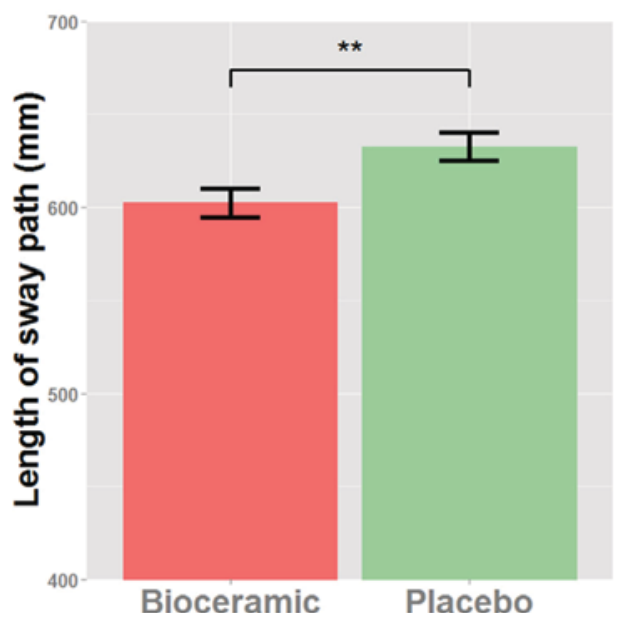

B

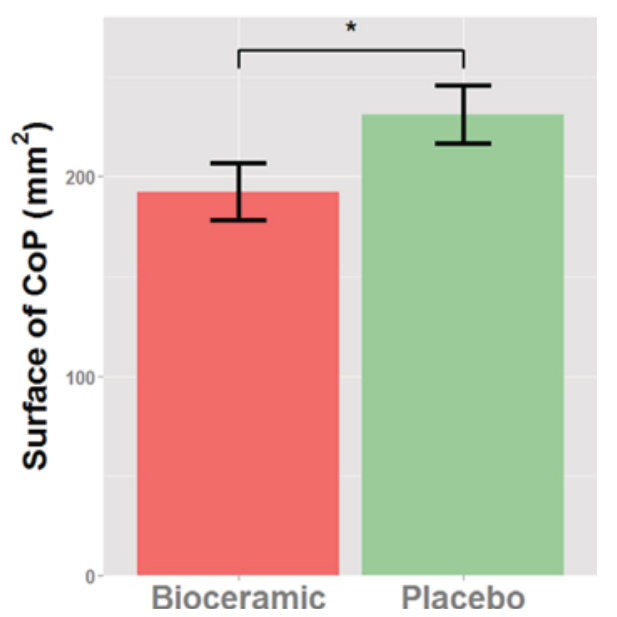

Fig. 2. Effect of the two types of suit on the length of the sway path (A - left panel) and the area of the $95 \%$ confidence ellipse (B - right panel) with standing subjects. In both A and $\mathrm{B}$, error bars represent the $95 \%$ confidence interval. 
A

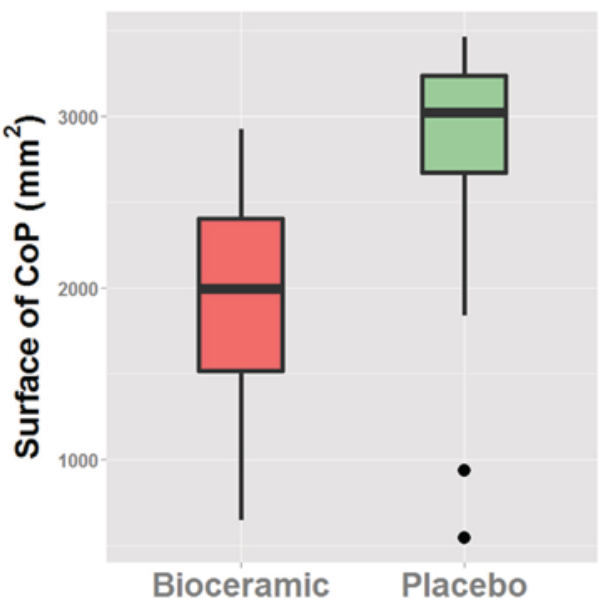

B

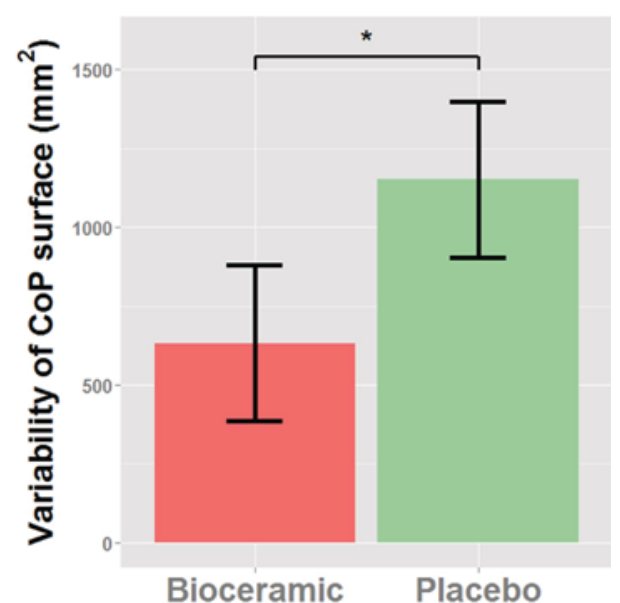

Fig. 3. Effect of the two types of suit on the area of the 95\% confidence ellipse (A - Left panel) and the intra-subject variability of surface of displacement (B - Right panel) with subjects holding a handstand. In $\mathrm{B}$, error bars represent the $95 \%$ confidence interval.

pattern was observed for 12 out of the 14 participants. As shown in Fig. 3B, the type of suit also affected significantly the individual (i.e., intra-subject) variability of the surface of displacements, $t(13)=-2.27, p=.0407, r=.53$. Specifically, variability was smaller when participants were wearing the bioceramic suit (mean standard deviation $=632 \mathrm{~mm}^{2}$ ) than when wearing the placebo suit ( mean standard deviation $=1152 \mathrm{~mm}^{2}$ ).

Range: As shown in Fig. 4A, the mean range of antero-posterior displacements of the CoP was significantly smaller with the bioceramic $(m=51.91 \mathrm{~mm})$ than with the placebo suit $(m=56.17 \mathrm{~mm}), t(13)=-2.278, p=.0403, r=.53$. The mean range of displacement along the left-right axis (Fig. 4B) was also significantly smaller with the bioceramic $(m=34.62 \mathrm{~mm})$ than with the placebo suit $(m=44.83 \mathrm{~mm}), t(13)=-2.432, p=.0302$, $r=.56$. On the other hand, the suit type did not affect individual range variability.

Velocity: The mean velocity of antero-posterior displacements of the CoP was not significantly different with the bioceramic $($ mean $=81.73)$ than with the placebo suit (mean $=83.67), t(13)=$ $-0.68, p=.506, r=.19$. The mean velocity of lateral displacements was significantly smaller with the bioceramic $(m=50.99)$ than with the placebo suit $(m=57.60), t(13)=-2.78, p=.0134, \quad r=.61$. Individual velocity variability was not affected by the suit type.

\section{Discussion}

Using a force platform to measure the displacements of the CoP, we tested the impact of bioceramic fabrics on postural control in (i) a standard standing postural task (Experiment 1) and (ii) a handstand stabilization task with expert gymnasts (Experiment 2).

Postural control was quantified by measuring the length, surface, range and velocity of displacement of the CoP. The first three variables (i.e., length, surface and range) constitute 'direct' indicators of postural control efficiency, whereas CoP velocity is supposed to be an indicator of muscular force variations, and therefore an 'indirect' indicator of postural control efficiency [11-13]. In Experiments 1 and 2, the surface and velocity of displacement of the CoP were significantly smaller when participants were wearing a bioceramic suit as compared to a placebo suit of same cut, size, appearance and elasticity. In the first experiment, with standing participants, the length of the sway path was also shorter with the bioceramic suit than with the
A

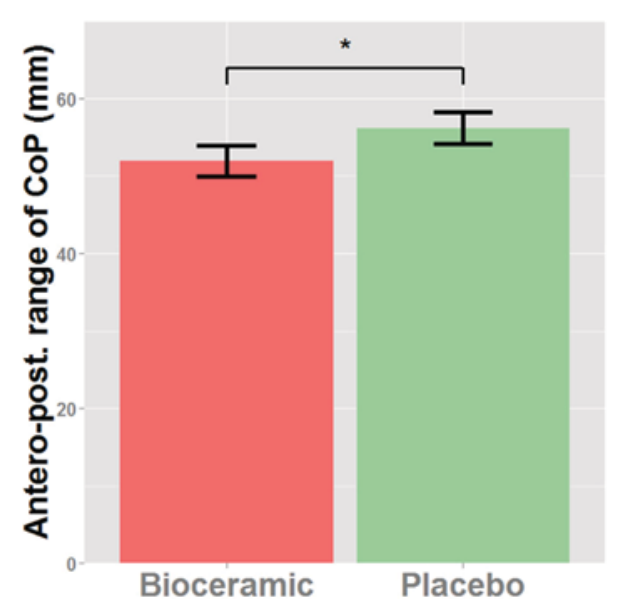

B

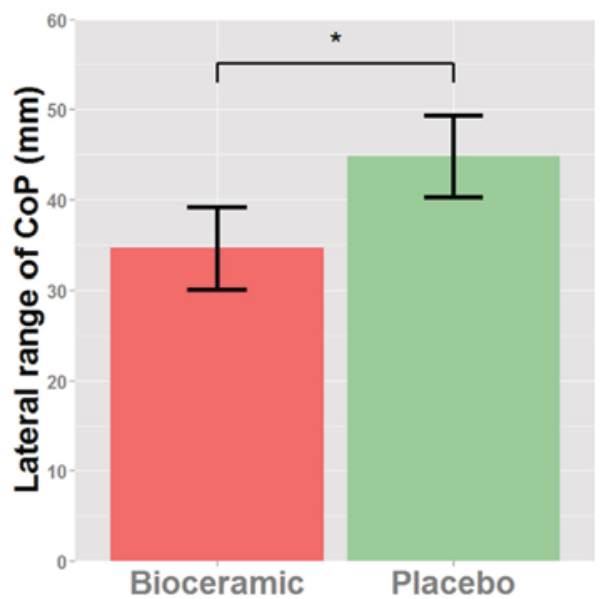

Fig. 4. Effect of the two types of suit on the mean range of displacement of the CoP along the antero-posterior axis (A - Left panel) and the left-right axis (B - Right panel) with subjects holding a handstand. In both $\mathrm{A}$ and $\mathrm{B}$, error bars represent the $95 \%$ confidence interval. 
placebo suit. These results show that the bioceramic suit helped participants improving their postural stability, whether standing on their feet or maintaining a handstand hold (for the gymnasts).

When participants were maintaining upright stance, wearing bioceramic garments resulted in a 5\% reduction of both the length of the sway path and the velocity of the displacements of the CoP in the antero-posterior direction. However, no significant difference was observed for the antero-posterior range which represents the amplitude of body motion along the antero-posterior axis. This suggests that when wearing the bioceramic suit, participants required less frequent corrections to achieve a similar postural stability.

In gymnastics, the handstand is estimated to be correct if the body segments are aligned and the body does not move, i.e., moves as little as possible. In that respect, CoP surface, which estimates the amplitude of body motion, appears to be the main parameter to discriminate gymnasts' performance. In our experiment, CoP surface was reduced by $27 \%$ when gymnasts wore bioceramic suits. In addition, individual variability of CoP surface was almost halved with respect to placebo. This indicates that bioceramic garments helped the gymnasts improving their stability and producing a 'stable' performance. Handstand stability was improved by bioceramic along both the lateral and anteroposterior axes, as evidenced by the observed reduced range. Interestingly, improved stability was observed for 12 out of the 14 gymnasts that participated in the experiment. The two gymnasts for whom the effect was not present were the gymnasts presenting the smallest $\mathrm{CoP}$ surface (irrespective of the suit), i.e., the most stable gymnasts. This suggests that bioceramic fabrics are most efficient when stability is impaired, which would also explain the much larger effects observed on the handstand hold than on the standard upright posture.

For postural regulation in the handstand as in erect posture, sensory perception is essential. In line with this, visual, somatosensory, and vestibular afferents are integrated for postural control [9,14-21], and stimulation of these sensory systems evokes changes in body sway. In our two experiments, the stimulation of these different sensory channels was similar across the two experimental conditions, namely with bioceramic and placebo suit. Therefore, the observed improvement in postural control is very unlikely to result from a difference in sensory stimulation or sensory weighting. Finally, upright stance and handstand are characterized by a body configuration that is unstable from a biomechanical perspective and requires continuous control by the neuromuscular system to maintain balance. It can be suggested that bioceramic materials could influence the process by which the body vary muscle contraction in immediate response to incoming information regarding external forces.

Even though physiological effects of bioceramic garments on posture remain unknown, our results show that it improves postural control in young non-athlete participants and expert gymnasts. Decline of balance function is considered one of the factors likely to be responsible for falls in a large percentage of older adults [22-24]. Indeed, as we age, balance function starts to decline and control of stance can become difficult for many older adults. It would therefore be useful to study if bioceramic garments could also improve balance stability in older adults and therefore reduce the likelihood of falls.

\section{Author contribution}

CC, MG and JPB conceived and designed the study, and wrote the article. CC, VG and JPB collected the data. All authors contributed to data analysis and interpretation. All authors revised the draft version of the article, and all approve the submitted version.

\section{Competing interests}

None of the authors have any conflicts of interests.

\section{Acknowledgement}

We thank HT Concept (http://www.htconcept.com/index.php/ presentation.html) for providing bioceramic and placebo suits.

\section{References}

[1] Leung TK, Kuo CH, Lee CM, Kan NW, Hou CW. Physiological effects of bioceramic material: harvard step, resting metabolic rate and treadmill running assessments. Chin J Physiol 2013;56(6):334-40. http://dx.doi.org/10.4077/ CJP.2013.BAB132.

[2] Vatansever F, Hamblin MR. Far infrared radiation (FIR): its biological effects and medical applications. Photonics Lasers Med 2012;4:255-66. http:/ dx.doi.org/10.1515/plm-2012-0034.

[3] Yoo HH, Kim YH, Cho HT, Shim HJ, Lee KB. Improvement of warmth retaining property of water vapor permeable/waterproof coated nylon fabric. J Korean Fiber Soc 1993:30(3):250-8.

[4] Yu SY, Chiu JH, Yang SD, Hsu YC, Lui WY, Wu CW. Biological effect of farinfrared therapy on increasing skin microcirculation in rats. Photodermatol Photoimmunol Photomed 2006;22(2):78-86. http://dx.doi.org/10.1111/j. 1600-0781.2006.00208.x.

[5] Ise N, Katsuura T, Kikuchi Y, Miwa E. Effect of far-infrared radiation on forearm skin blood flow. Ann Physiol Anthropol 1987;6(1):31-2.

[6] Ko GD, Berbrayer D. Effect of ceramic-impregnated thermoflow gloves on patients with Raynaud's syndrome: randomized, placebo-controlled study. Altern Med Rev 2002;7(4):328-35.

[7] Katsuura T, Fukuda S, Okada A, Kikuchi Y. Effect of ceramic-coated clothing on forearm blood flow during exercise in a cool environment. Ann Physiol Anthropol 1989;8(1):53-5.

[8] Leung TK, Lee CM, Tsai SY, Chen YC, Chao JS. A pilot study of ceramic powder far-infrared ray irradiation (cFIR) on physiology: observation of cell cultures and amphibian skeletal muscle. Chin J Physiol 2011;54(4):247-54. http:// dx.doi.org/10.4077/CJP.2011.AMM044.

[9] Massion J. Postural control system. Curr Opin Neurobiol 1994;4(6):877-87.

[10] Schubert P, Kirchner M, Schmidtbleicher D, Haas CT. About the structure of posturography: sampling duration, parametrization, focus of attention. J Biomed Sci Eng 2012;5:496-507.

[11] Asseman F, Caron O, Cremieux J. Is there a transfer of postural ability from specific to unspecific postures in elite gymnasts? Neurosci Lett 2004;358(2): 83-6. http://dx.doi.org/10.1016/j.neulet.2003.12.102.

[12] Asseman FB, Caron O, Cremieux J. Are there specific conditions for which expertise in gymnastics could have an effect on postural control and performance? Gait Posture 2008;27(1):76-81. http://dx.doi.org/10.1016/j.gaitpost. 2007.01.004.

[13] Ruhe A, Fejer R, Walker B. The test-retest reliability of centre of pressure measures in bipedal static task conditions - a systematic review of the literature. Gait Posture 2010;32(4):436-45. http://dx.doi.org/10.1016/j.gaitpost.2010.09.012.

[14] Bronstein AM, Guerraz M. Visual-vestibular control of posture and gait: physiological mechanisms and disorders. Curr Opin Neurol 1999;12(1):5-11.

[15] Fitzpatrick RC, Day BL. Probing the human vestibular system with galvanic stimulation. J Appl Physiol (1985) 2004;96(6):2301-16. http://dx.doi.org/ 10.1152/japplphysiol.00008.2004.

[16] Guerraz M, Bronstein AM. Mechanisms underlying visually induced body sway. Neurosci Lett 2008;443(1):12-6. http://dx.doi.org/10.1016/j.neulet.2008.07.053

[17] Kavounoudias A, Roll R, Roll JP. Foot sole and ankle muscle inputs contribute jointly to human erect posture regulation. J Physiol 2001;532(Pt 3):869L 878 .

[18] Lackner JR, DiZio P, Jeka J, Horak F, Krebs D, Rabin E. Precision contact of the fingertip reduces postural sway of individuals with bilateral vestibular loss. Exp Brain Res 1999;126(4):459-66.

[19] Lee DN, Lishman JR. Vision - most efficient source of proprioceptive information for balance control. Agressologie 1977;18:83-94.

[20] Nashner LM, Black FO, Wall 3rd C. Adaptation to altered support and visual conditions during stance: patients with vestibular deficits. J Neurosci 1982;2(5):536-44

[21] Rabin E, Bortolami SB, DiZio P, Lackner JR. Haptic stabilization of posture: changes in arm proprioception and cutaneous feedback for different arm orientations. J Neurophysiol 1999;82(6):3541-9.

[22] Shumway-Cook A, Brauer S, Woollacott M. Predicting the probability for falls in community-dwelling older adults using the Timed Up \& Go Test. Phys Ther 2000;80(9):896-903.

[23] Shumway-Cook A, Woollacott M. Attentional demands and postural control: the effect of sensory context J Gerontol A Biol Sci Med Sci 2000·55(1):M10-6.

[24] Tinetti ME, Williams CS. The effect of falls and fall injuries on functioning in community-dwelling older persons. J Gerontol A Biol Sci Med Sci 1998; 53(2):M112-9. 